\title{
A Non-damaging Method to Analyze the Configuration and Dynamics of Nitrotyrosines in Proteins
}

Irene Díaz-Moreno, ${ }^{*[a]}$ Pedro M. Nieto, ${ }^{[b]}$ Rebecca Del Conte, ${ }^{[c]}$ Margarida Gairí, ${ }^{[d]}$ José M. García-Heredia, ${ }^{[a]}$ Miguel A. De la Rosa, ${ }^{[a]}$ and Antonio Díaz-Quintana ${ }^{[a]}$

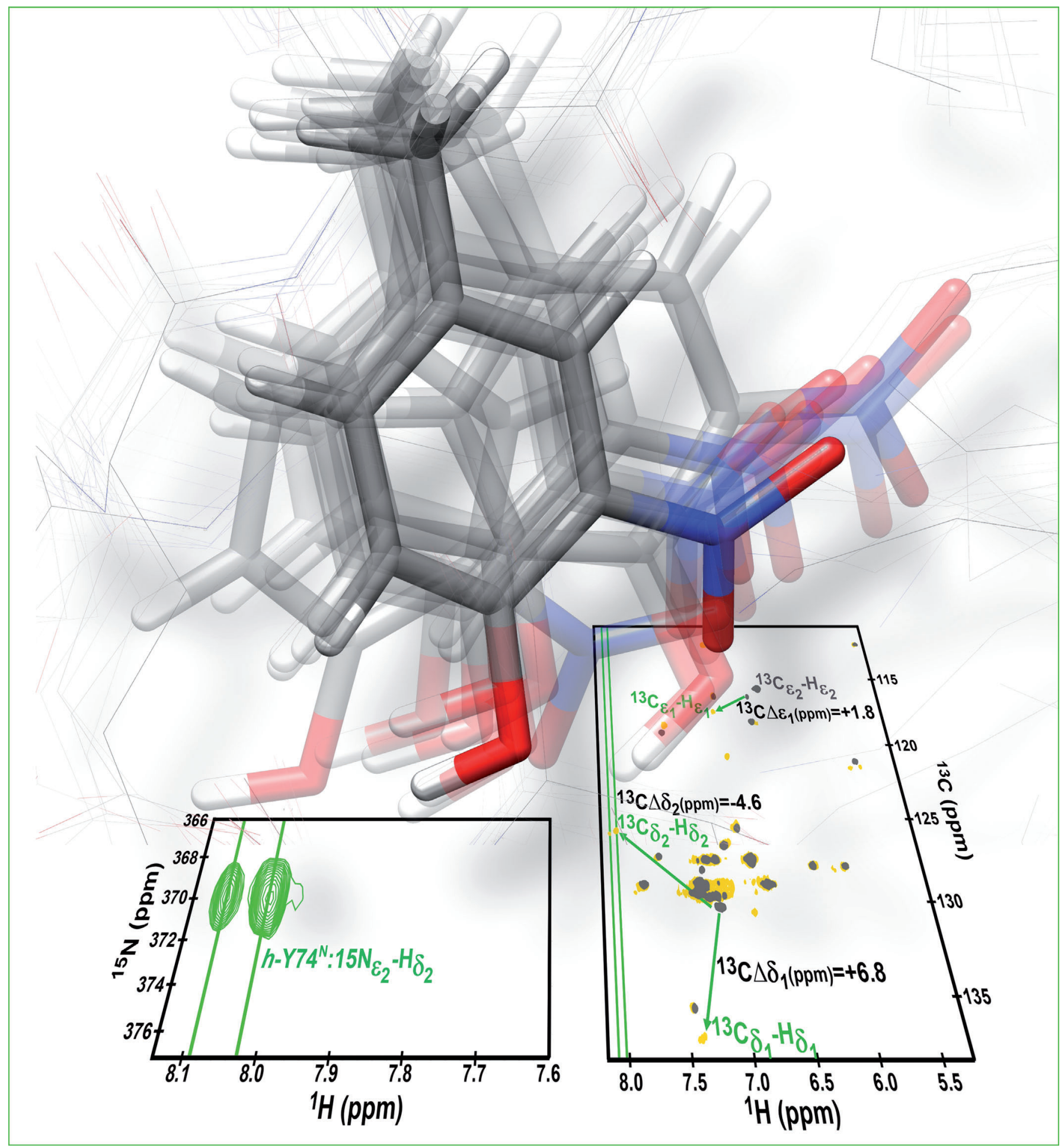




\begin{abstract}
Often, deregulation of protein activity and turnover by tyrosine nitration drives cells toward pathogenesis. Hence, understanding how the nitration of a protein affects both its function and stability is of outstanding interest. Nowadays, most of the in vitro analyses of nitrated proteins rely on chemical treatment of native proteins with an excess of a chemical reagent. One such reagent, peroxynitrite, stands out for its biological relevance. However, given the excess of the nitrating reagent, the resulting in vitro modifica-
\end{abstract}

tion could differ from the physiological nitration. Here, we determine unequivocally the configuration of distinct nitrated-tyrosine rings in single-tyrosine mutants of cytochrome $c$. We aimed to confirm the nitration position by a non-destructive method. Thus, we have resorted to ${ }^{1} \mathrm{H}-{ }^{15} \mathrm{~N}$ heteronuclear single

Keywords: configuration determination $\cdot$ molecular dynamics $\cdot$ nitrotyrosine • NMR spectroscopy . proteins quantum coherence(HSQC) spectra to identify the ${ }^{3} J(\mathrm{~N}-\mathrm{H})$ correlation between a ${ }^{15} \mathrm{~N}$-tagged nitro group and the adjacent aromatic proton. Once the chemical shift of this proton was determined, we compared the ${ }^{1} \mathrm{H}-{ }^{13} \mathrm{C}$ HSQC spectra of untreated and nitrated samples. All tyrosines were nitrated at $\varepsilon$ positions, in agreement to previous analysis by indirect techniques. Notably, the various nitrotyrosine residues show a different dynamic behaviour that is consistent with molecular dynamics computations.

\section{Introduction}

Nitration of tyrosine residues is associated to more than 60 human disorders. ${ }^{[1-3]}$ Diverse agents can induce such a posttranslational protein modification that often leads to changes of the catalytic activity of the enzyme, its turnover or its localization..$^{[1,2,4,5]}$ In many cases, tyrosine nitration interferes with the phosphorylation of the residue, thereby becoming a regulation switch. ${ }^{[5,6]}$ In a cell, the accumulation of nitrated proteins results from increased generation of reactive nitrogen/oxygen species (RNOS), which causes impairment in either the proteasome ${ }^{[7]}$ or the denitration system. ${ }^{[3]}$

The reactivity of tyrosine residues towards nitrating agents, such as peroxynitrite, depends on several factors. ${ }^{[8,9]}$ For instance, it is unrelated to any protein sequence pattern, but charged residues next to the target affect its reactivity.

[a] Dr. I. Díaz-Moreno, Dr. J. M. García-Heredia,

Prof. M. A. De la Rosa, Dr. A. Díaz-Quintana

Instituto de Bioquímica Vegetal y Fotosíntesis

Centro de Investigaciones Científicas Isla de la Cartuja,

Universidad de Sevilla-C.S.I.C.

Avda. Américo Vespucio, 49, 41092 Sevilla (Spain)

Fax: $(+34) 954460065$

E-mail: idiazmoreno@us.es

[b] Dr. P. M. Nieto

Instituto de Investigaciones Químicas

Centro de Investigaciones Científicas Isla de la Cartuja,

Universidad de Sevilla-C.S.I.C.

Avda. Américo Vespucio, 49, 41092 Sevilla (Spain)

[c] Dr. R. Del Conte

CERM

University of Florence

Via Luigi Sacconi 6, 50019 Sesto Fiorentino Florence (Italy)

[d] Dr. M. Gairí

NMR Facility, Centres Científics i Tecnològics

Universitat de Barcelona

Parc Científic de Barcelona

Baldiri Reixac, 20, 08028 Barcelona (Spain)

Supporting information for this article is available on the WWW

under http://dx.doi.org/10.1002/chem.201103413.
In addition, the selectivity of a nitrating substance depends on its chemical nature. The target amino acid must be accessible to the reagent. In fact, most nitrated tyrosine residues locate in loop regions, and the presence of turn-inducing residues near a tyrosine favours its modification. Finally, there is a competition between alternative targets nearby, including sulfhydryl groups from cysteine residues.

As pointed out by Ischiropoulos, ${ }^{[4]}$ the in vivo relevance of Tyr modification was controversial until peroxynitrite was shown to be a physiological nitration agent. Peroxynitrite $\left(\mathrm{ONOO}^{-}\right)$originates under nitro-oxidative stress, being the mitochondria one of its major sources within the cell. Notably, its tyrosine-nitrating activity is optimum at neutral $\mathrm{pH} .{ }^{[9]}$ Thus, recent reports on the effects of protein nitration rely on the treatment of isolated proteins with this chemical. ${ }^{[10-16]}$

In general, phenol rings are preferentially nitrated at ortho positions ( $\varepsilon$ carbons in Tyr residues) relative to the hydroxyl group. Still, nitration could also take place at meta carbons, although the yield in this case is lower. In fact, under acidic conditions, the nitration of cresol, an analogous to tyrosine, occurs in both, ortho- and meta positions in a 3:1 ratio. ${ }^{[17]}$ However, tyrosine nitration seems to occur exclusively at the ortho position, both in vitro and in vivo, upon treatment with tetranitromethane. ${ }^{[18]}$ In that report, the configuration of the nitrotyrosine side chain was tested by gas chromatography coupled to mass spectrometry. Recently, a new methodology for nitrotyrosine detection and quantification has been developed. ${ }^{[19-21]}$ Nevertheless, the analysis of tyrosine nitration by mass spectrometry remains challenging nowadays for many proteins. ${ }^{[20]}$

Given the heterogeneity of the environment around the distinct tyrosine residues within a protein, the nitration efficiencies at $\varepsilon$ - and $\delta$ positions could vary from one residue to another. To study the effect of Tyr nitration of proteins in vitro, a large excess of this chemical is usually added to protein samples. These facts could originate heterogeneity of the nitrated sample, thereby biasing the analysis. In addition, haem-containing proteins are able to reducing the nitro group to amine. ${ }^{[22]}$ 
Hence, we report herein a suitable, non-destructive method to determine and assign the nitration site configuration based on NMR spectroscopy. This approach consists of using both ${ }^{1} \mathrm{H}-{ }^{15} \mathrm{~N}$ and ${ }^{1} \mathrm{H}-{ }^{13} \mathrm{C}$ heteronuclear experiments. In a first step, the ${ }^{3} J(\mathrm{~N}-\mathrm{H})$ scalar coupling between the nitro group and the nearby aromatic hydrogen is detected as a correlation in a ${ }^{1} \mathrm{H}-{ }^{15} \mathrm{~N}$ HSQC, instead of the conventional ${ }^{1} J(\mathrm{~N}-\mathrm{H})$. This requires an increase in the delays in the INEPT $^{[23]}$ module of the pulse sequence to allow the magnetization transfer through this weaker coupling. In a second step, the nitration configuration of the ring is determined by analyzing the ${ }^{1} \mathrm{H}$ - and ${ }^{13} \mathrm{C}$ chemical shifts from aromatic proton-carbon correlations in the ${ }^{1} \mathrm{H}-{ }^{13} \mathrm{C}$ HSQC spectra, capitalizing on the lower sensitivity of the ${ }^{13} \mathrm{C}$ chemical shifts $(\delta)$ to changes within the molecular environment.

As a case study, we analyzed the different products resulting from nitration of the distinct tyrosine residues of human cytochrome $c(\mathrm{Cc})$ by peroxynitrite. Nitration of this protein affects its stability and the chemical properties of its haem moiety. ${ }^{[13,16]}$ In addition, such modifications bias the binding of $\mathrm{C} c$ to Apaf- $1,{ }^{[16]}$ thereby impairing the activation of the caspase cascade. ${ }^{[24]}$ These effects strongly depend on the location of the nitrated residue. For instance, we have recently shown that nitration at the positions 46 and 48 lead to specific in vivo degradation. ${ }^{[25]}$ We show that most tyrosines are unequivocally nitrated at the $\varepsilon$ position. Still, we failed for Tyr67, whose signals are broadened beyond detection, probably because of its proximity to the haem group. Notably, nitration affects the dynamics of the target residue in a different manner, depending on its location within the structure.

\section{Results}

As pointed out before, the aim of this work is to provide an NMR-based approach to test the effects of treating tyrosine residues with peroxynitrite, by using $h-\mathrm{C} c$ as a model. This protein contains five tyrosine residues (see Figure S1 in Supporting Information) located at positions 46, 48, 67, 74 and 97 of the peptide sequence. To get nitration of any specific tyro- sine residue, we used single-tyrosine mutants, in which all tyrosines but the targeted one were replaced by $\mathrm{Phe}$, as in previous reports. ${ }^{[1,16,25]}$ The tags of these mutants are $h$-Y46, $h$ Y48, $h$-Y67, $h$-Y74 and $h$-Y97. Their nitrated forms are named by adding the " ${ }_{\mathrm{N}}$ " subscript tag; for example, $h-\mathrm{Y} 48_{\mathrm{N}}$.

To prove whether a given tyrosine residue is nitrated at its $\delta$ - or $\varepsilon$ position, the information interpreted from the heteronuclear ${ }^{1} \mathrm{H}^{15} \mathrm{~N}$ and ${ }^{1} \mathrm{H}^{13} \mathrm{C}$ experiments was combined. Thus, we acquired a ${ }^{1} \mathrm{H}_{-}{ }^{15} \mathrm{~N}$ HSQC (Figure 1, left panels) to determine the frequency of the proton next to the nitration point, as revealed by the corresponding ${ }^{3} \mathrm{~J}(\mathrm{H}-\mathrm{N})$ correlation cross-peak. Also, we took advantage of the ${ }^{13} \mathrm{C}$ chemical shift differences among $\mathrm{C}_{\delta}$ and $\mathrm{C}_{\varepsilon}$ in the aromatic rings of Tyr, with the $C_{\varepsilon}$ resonating at higher field than the $C_{\delta}$. Thus, we acquired ${ }^{1} \mathrm{H}_{-}{ }^{13} \mathrm{C}$ HSQC spectra for both non-nitrated and nitrated samples and compared them to identify the protoncarbon aromatic correlations corresponding to Tyr and Tyr$\mathrm{NO}_{2}$, respectively (Figure 1, right panels). In the spectra of the non-nitrated sample, the chemical shifts of the carbon
A
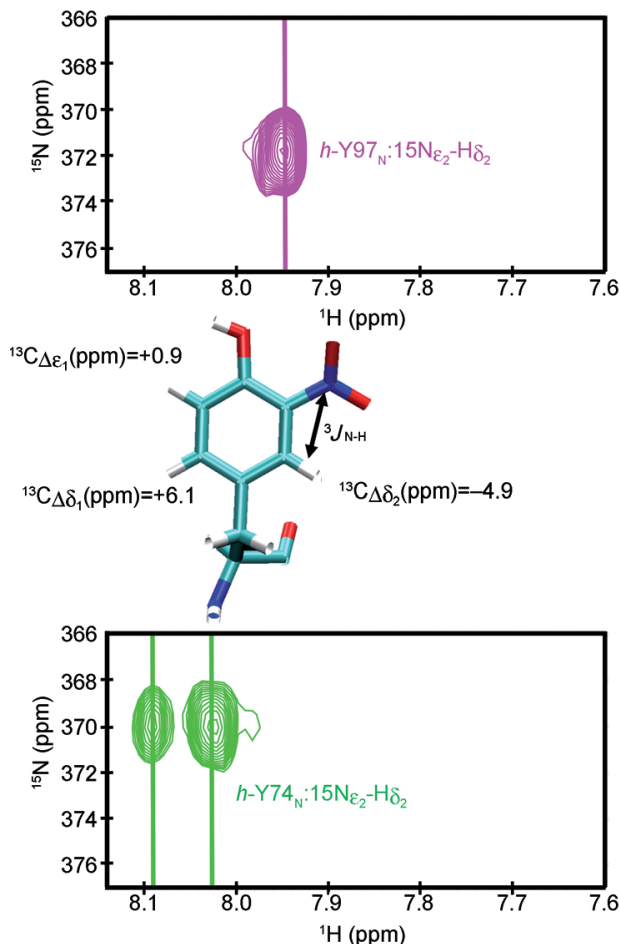

Figure 1. Determination of the nitration position in Tyr97 and Tyr74 of Cc: The ${ }^{1} \mathrm{H}_{-}{ }^{15} \mathrm{~N}-\mathrm{HSQC}$ spectra of the nitrated species $\mathrm{Y} 97_{\mathrm{N}}(\mathrm{A})$ and $\mathrm{Y} 74_{\mathrm{N}}$ (B) are shown on the left. Below in panel A, the correlation observed in the ${ }^{1} \mathrm{H}-{ }^{15} \mathrm{~N}$ HSQC spectra is marked by a double arrow in a molecular model. The model also shows expected chemical shift changes of carbon and upon a nitration in the ortho position, according to Hansen. ${ }^{[34]}$ Right: the ${ }^{1} \mathrm{H}_{-}{ }^{13} \mathrm{C}$ HSQC spectra of untreated samples (in grey, Y97 in A, Y74 in B) are overlaid with those (yellow) of their respective nitrated forms. Arrows indicate the perturbation of the signal of the remaining protons of the aromatic ring upon nitration. 
atoms at positions $\delta$ and $\varepsilon$ of the single-Tyr residue lay at different regions in the spectrum. Moreover, their frequencies are much less influenced by changes in the molecular environment than proton frequencies. Furthermore, there is no overlap between the $\mathrm{H}-\mathrm{C}_{\varepsilon}$ correlation resonances and the signals corresponding from phenylalanine residues. Upon nitration, the signal of the replaced proton disappears and those of the rest of protons of the aromatic ring undergo a substantial shift. This allowed us to assign the signals from the tyrosine residue unequivocally. We can then identify the nitration site because we know the frequency of the closest proton, and to what extent the carbon frequencies shift, as illustrated in Figure 1. In fact, for small organic molecules, the carbon at the ortho position regarding the nitrated one shifts $\delta=4.9 \mathrm{ppm}$ upfield, and those at meta- and para positions shift $\delta=0.9$ and $6.1 \mathrm{ppm}$ downfield, respectively. ${ }^{[34]}$

Figure $1 \mathrm{~A}$ shows the effect of nitration on the spectra of the reduced $h$-Y97 mutant. Upon nitration, a ${ }^{15} \mathrm{~N}-{ }^{1} \mathrm{H}$ correlation $\left(\mathrm{N}_{\varepsilon_{2}}-\mathrm{H}_{\delta_{2}}\right)$ appears in the HSQC at $\delta=372 \mathrm{ppm}$ in the ${ }^{15} \mathrm{~N}$ dimension, and three signals are shifted in the ${ }^{1} \mathrm{H}-{ }^{13} \mathrm{C}$ HSQC with respect to the spectrum of the untreated sample. One of them shows the proton frequency $(\delta=$ $7.94 \mathrm{ppm}$ ) already observed in the ${ }^{1} \mathrm{H}-{ }^{15} \mathrm{~N}$ HSQC, being assigned to the proton next to the nitration point. We expect an upfield shift in the carbon frequency at this position and the shifted signal lies around $\delta=10 \mathrm{ppm}$ downfield of the peaks corresponding to the remaining epsilon carbons. In addition, a $\mathrm{C}_{\varepsilon_{1}}-\mathrm{H}_{\varepsilon_{1}}$ correlation is displaced downfield approximately $\delta=1 \mathrm{ppm}$ compared with its equivalent in the untreated sample. Hence, we can assign unequivocally the signal at $\delta=7.94 \mathrm{ppm}$ to one of the $\delta$ hydrogens $\left(\mathrm{C}_{\delta_{2}}-\mathrm{H}_{\delta_{2}}\right.$ correlation) instead of a $\mathrm{C}_{\varepsilon}-\mathrm{H}_{\varepsilon}$ correlation. Moreover, no signal below $\delta=123 \mathrm{ppm}$ in the ${ }^{13} \mathrm{C}$ dimension shifts upfield, thereby indicating that nitration of $\mathrm{Y} 97$ is restricted to its $\varepsilon$ carbons. Finally, a $\mathrm{C}_{\delta_{1}}-\mathrm{H}_{\delta_{1}}$ correlation shows the expected $\delta=6 \mathrm{ppm}$ downfield shift for a carbon at the para position. Further assignment of aromatic nuclei was achieved by comparing the spectra of $h$-WT and its single-tyrosine mutants. Upon Tyr97 nitration, the cross peak corresponding to the $\mathrm{H}_{\eta_{2}}$ of $\operatorname{Trp59}\left({ }^{1} \mathrm{H}\right.$ signal at $\left.\delta=5.6 \mathrm{ppm}\right)$ shows significant chemical shift variations.

The nitration of the reduced $h$-Y74 mutant yielded a similar pattern (Figure 1B). Three signals in the ${ }^{1} \mathrm{H}-{ }^{13} \mathrm{C}-\mathrm{HSQC}$ undergo shifts larger than $\delta=1.0 \mathrm{ppm}$, besides several peaks showing small perturbations. Again, our results indicate that Tyr74 is nitrated at a $\varepsilon$ carbon by peroxynitrite. However, the splitting of the $\mathrm{H}_{\delta_{2}}$ signal in the two NMR spectra indicates that the nitrated tyrosine residue undergoes a slow chemical-exchange regime in the millisecond timescale. In addition, a signal with $\delta_{\mathrm{H}}=5.7 \mathrm{ppm}$ and $\delta_{\mathrm{C}}=121.1 \mathrm{ppm}$ in the spectra of the untreated sample splits upon nitration. The ${ }^{13} \mathrm{C}$ frequency can be assigned to either $\mathrm{C}_{5}$ or $\mathrm{C}_{6}$ of an indole ring. That is, to either $\mathrm{C}_{\zeta_{3}}$ or $\mathrm{C}_{\eta_{2}}$ of Trp59. This agrees with the assignment of the ${ }^{1} \mathrm{H}$ signal at $\delta=5.7 \mathrm{ppm}$ to $\mathrm{H}_{\eta_{2}}$ of Trp59. ${ }^{[35]}$ Hence, the conformation exchange affects the orientation of Tyr74 with respect to the nearby indole ring of Trp59. Moreover, the ${ }^{1} \mathrm{H}-{ }^{13} \mathrm{C}-\mathrm{HSQC}$ shows many new signals at the region of aromatic $\mathrm{C}_{\delta}-\mathrm{H}_{\delta}$. This may be attributed to the conformational exchange of Tyr74 affecting to Tyr67. In fact, Tyr67 contacts both Tyr74 and Trp59 (see Figure S2 in the Supporting Information). Interestingly, another cross peak at $\delta=120.5 \mathrm{ppm}$ in ${ }^{13} \mathrm{C}$ and $\delta=6.8 \mathrm{ppm}$ in ${ }^{1} \mathrm{H}$ appears in the nitrated $h$-Y74 mutant spectrum.

The effects of nitration on the NMR spectra of oxidized $h$-Y46 and $h$-Y48 single-tyrosine mutants are illustrated in Figure 2. The ${ }^{1} \mathrm{H}^{-15} \mathrm{~N}$ HSQC region in Figure $2 \mathrm{~A}$ shows the ${ }^{3} J$ correlation between the ${ }^{15} \mathrm{~N}$ from the nitro group and the adjacent aromatic proton. Notably, the side-chain ring of nitro-Tyr 48 shows a restricted mobility. In fact, we detect a major population with a $\delta\left(\mathrm{H}_{\delta_{2}}\right)$ value of $7.8 \mathrm{ppm}$, and a minor one at $\delta=7.75 \mathrm{ppm}$. The two conformations exhibit a slow exchange in the millisecond timescale. The assignment can be corroborated by the appearance of a correlation of these chemical shifts with that $(\delta=126.5 \mathrm{ppm})$ expected for $\mathrm{C}_{\delta_{2}}$ in a ring nitrated at $\mathrm{C}_{\varepsilon_{2}}$ in the ${ }^{1} \mathrm{H}-{ }^{13} \mathrm{C}$ HSQC spectra. Notably, $\mathrm{Y} 48$ is mostly buried in the protein, thereby explaining the reduced mobility of the nitrated residue.

In contrast to Y48, Y46 and its nitrated form are more exposed to solvent and have larger mobilities. Figure 2 B shows how the signal corresponding to the ${ }^{3} J\left(\mathrm{~N}_{\varepsilon_{2}}-\mathrm{H}_{\delta_{2}}\right)$ correlation indicates a multiple conformation exchange of the molecule. In fact, the three proton frequencies exhibit the ${ }^{3} J\left(\mathrm{~N}_{\varepsilon_{2}}-\mathrm{H}\right)$ correlation, and all correlate with a single carbon chemical shift $(\delta=126.5 \mathrm{ppm})$ that can be assigned to $\mathrm{C}_{\delta_{2}}$. The larger mobility of this residue, as compared with that of $\mathrm{Y} 48$, is clearly illustrated by the data from MD computations displayed in Figure 4.

Both oxidized $h$-Y46 and $h$-Y48 single-tyrosine mutants show ${ }^{1} \mathrm{H}^{13} \mathrm{C}$ HSQC spectra that are quite different from those of the previous mutants because $\mathrm{Cc}$ becomes a highspin species when it is nitrated at positions 46 and $48 .{ }^{[25]}$

Y67 is the only tyrosine residue lying on top of the haem group; the aromatic ring of which is buried inside the protein. However, it is nitrated with high efficiency in the presence of peroxynitrite. ${ }^{[11,14,16,26]}$ Notably, as shown in Figure $3 \mathrm{~A}$, we were unable to find the ${ }^{3} J\left(\mathrm{~N}_{\varepsilon_{2}}-\mathrm{H}_{\delta_{2}}\right)$ correlation in the ${ }^{1} \mathrm{H}-{ }^{15} \mathrm{~N}$ HSQC spectra of the $h-\mathrm{Y} 67_{\mathrm{N}}$ form. We changed the spectral window to search alternative functional groups containing nitrogen, including amines that result from reduction of the nitro group. In fact, reduction of nitro groups to amine groups has already been reported for haem proteins. ${ }^{[12]}$ Still, we were unable to finding any correlation signal. Even though MALDI-TOF analysis (Figure 3B, left panel) showed a mass difference of $(47 \pm 3)$ Da between $h$ Y 67 and $h-\mathrm{Y} 67_{\mathrm{N}}$, which is consistent with a replacement of a hydrogen atom by a nitro group. Furthermore, $h-\mathrm{Y} 67_{\mathrm{N}}$ bound a polyclonal antibody against 3-nitrotyrosine (Figure $3 \mathrm{~B}$, right panel), whereas untreated samples were unable to. Notably, the antibody does not cross-react with the aminotyrosine, according to the manufacture instructions. Moreover, the protein showed no fluorescence at $308 \mathrm{~nm}$, as expected from a putative presence of aminotyrosine. ${ }^{[36]}$ 
A
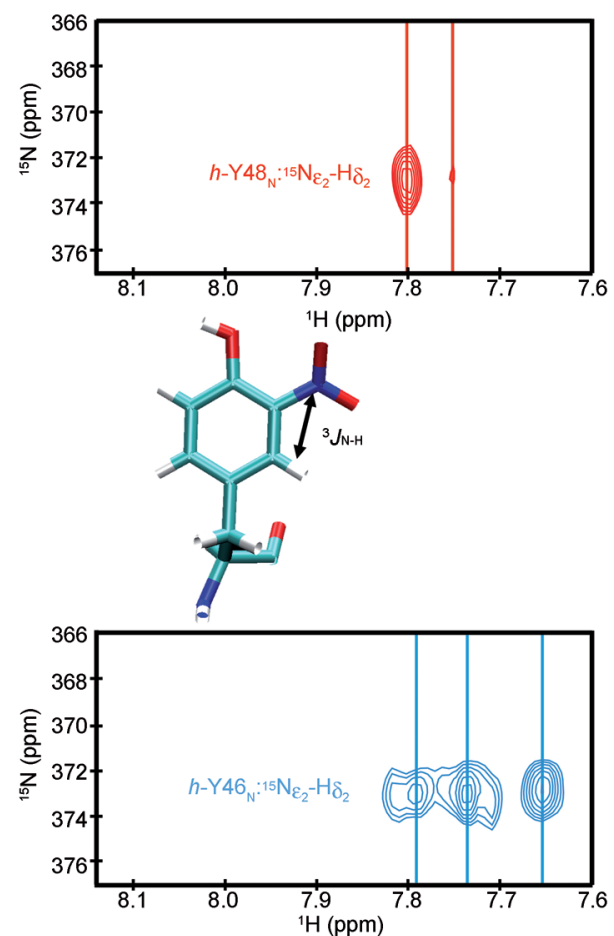

B
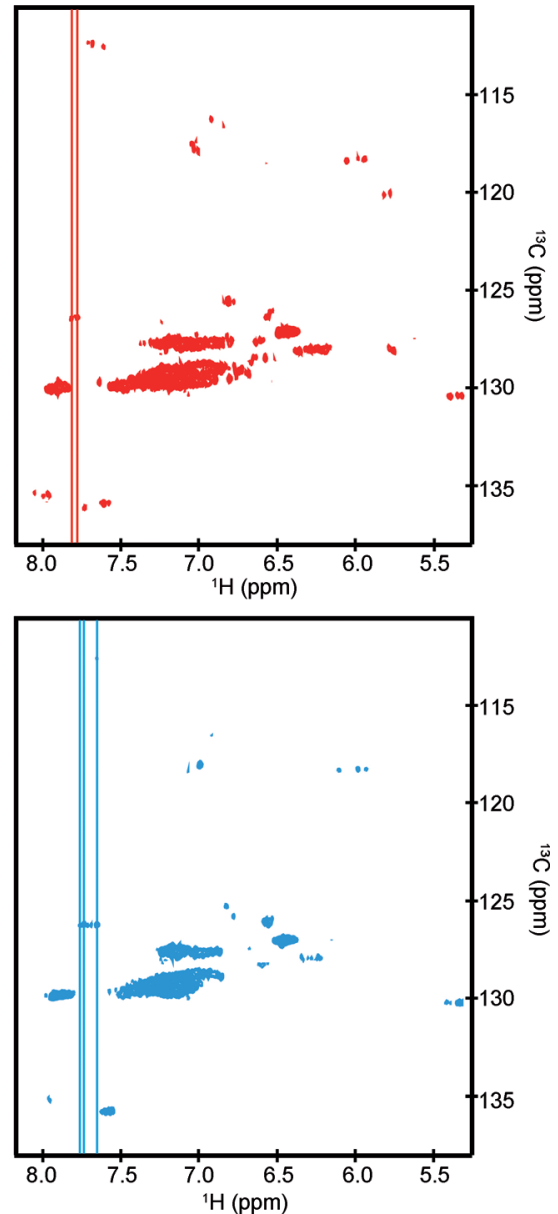

Figure 2. Determination of the nitration position in Tyr48 and Tyr46: As in Figure 1, the ${ }^{1} \mathrm{H}-{ }^{15} \mathrm{~N}-\mathrm{HSQC}$ spectra of the nitrated species $\mathrm{Y} 48_{\mathrm{N}}(\mathrm{A})$ and $\mathrm{Y} 46_{\mathrm{N}}$ (B) are shown on the left. The correlation observed in the ${ }^{1} \mathrm{H}-{ }^{15} \mathrm{~N}$ HSQC spectra is marked by a double arrow in a molecular model. Right: the ${ }^{1} \mathrm{H}-{ }^{13} \mathrm{C}$ HSQC spectra of treated samples $\left(\mathrm{Y}_{4} 8_{\mathrm{N}}\right.$ in $\mathrm{A}$; $\mathrm{Y}_{4} 6_{\mathrm{N}}$ in $\left.\mathrm{B}\right)$ are shown.
As it was noted before, some of the analyzed resonances exhibit a slow exchange regime, whereas others do not. Notably, the residues showing that behaviour, namely Y46 and Y74, are more exposed to solvent. Hence, we tested the effect of nitration on the side-chain mobility in the context of the residue location by MD computations. Figure 4 shows the distribution of the dihedral angles for the two rotatable bonds of the different tyrosine sidechains in native and singly nitrated samples. Notably, the $\mathrm{C}_{\alpha}{ }^{-}$ $\mathrm{C}_{\beta}$ bonds $\left(\chi_{1}\right.$ angles) seem to be equally restrained in all the tyrosine residues of the untreated protein. In fact, fitting the angle frequencies to a Gaussian distribution allows to determining a harmonic force constant, which is similar (see Table S1 in the Supporting Information) for all residues. Still, Tyr46 is somewhat more mobile. Average $\chi_{1}$ values lie between 170 and 195 degrees, with the exception of the residue corresponding to Tyr48, which shows a gauche conformation. Upon nitration, the $\mathrm{C}_{\alpha}-\mathrm{C}_{\beta}$ bonds of residues 48 ,

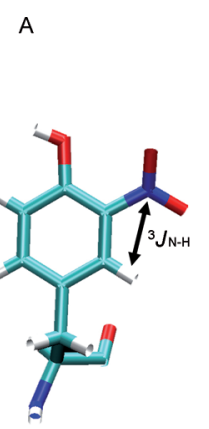

B
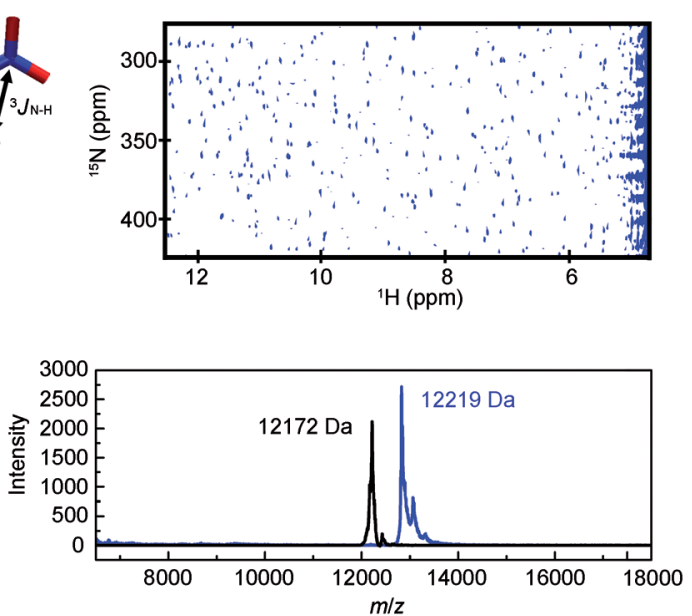

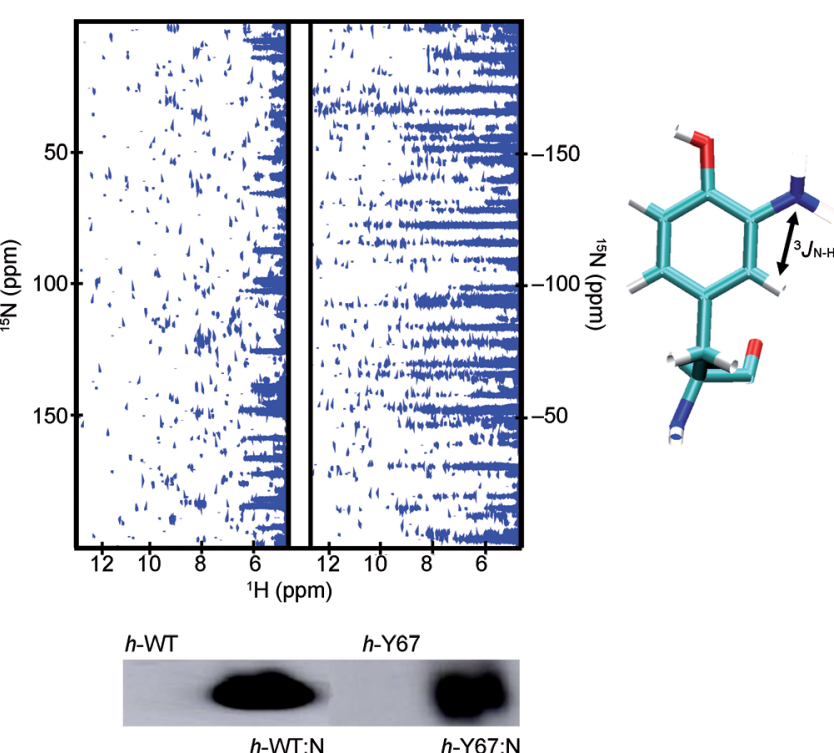

Figure 3. Effects of nitration on the NMR spectra of Tyr67: A) ${ }^{1} \mathrm{H}^{15} \mathrm{~N}$ HSQC spectra of the Y67 species. Contours were set at the noise level to enhance any broad signal in the spectra. Left, the spectral window was set to scan the region corresponding to nitro groups, as in Figure 1 and 2 . The spectra on the right scan the frequency range typical of amines and nitrate, according to Mason. ${ }^{[37]} \mathrm{B}$ ) Left panel shows MALDI-TOF spectra of $h$-Y67 (black) and $h$-Y67 $($ blue). Right panel shows Western blot analysis of $h$-WT, $h$-WT,$h$-Y67 and $h$-Y67 , using a 3-nitrotyrosine-specific antibody. 

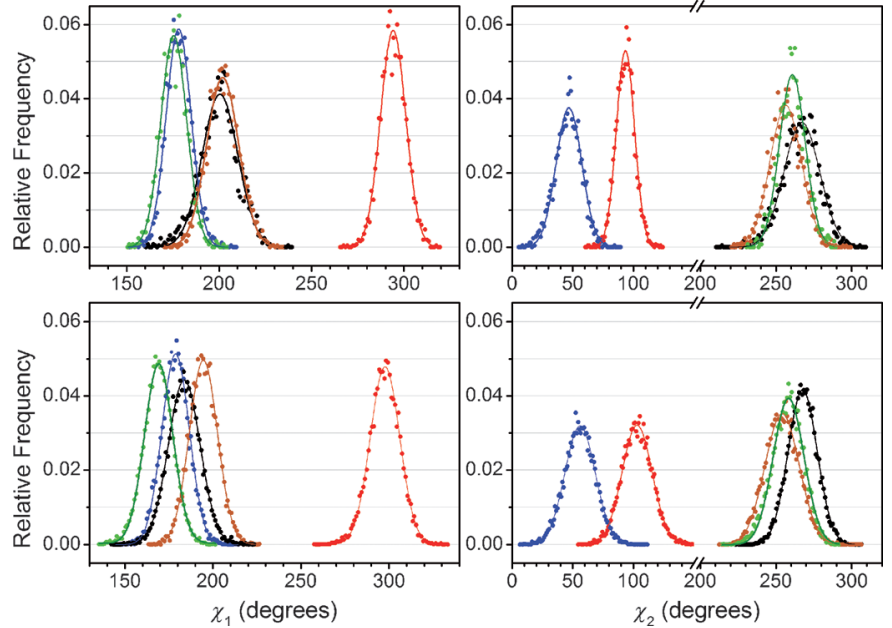

Figure 4. Conformations of tyrosine residues sampled along MD computations: Upper: distribution of $\chi_{1}$ (left) and $\chi_{2}$ (right) in nitrated human $\mathrm{C} c$ tyrosine residues. Lines represent fits of the data to Gaussian functions. Lower: distribution of the above dihedral angles in untreated samples. Data corresponding to Y46 are in black, Y48 in red, Y67 in blue, Y74 in brown and Y97 in green.

67 and 97 are more constrained by the surrounding atoms, whereas the side chains of Tyr46 and Tyr 74 slightly become more mobile.

As expected, Figure 4 shows that rotation around the $\mathrm{C}_{\beta^{-}}$ $\mathrm{C}_{\gamma}$ bonds $\left(\chi_{2}\right.$ angles $)$ is better allowed than around $\mathrm{C}_{\alpha}-\mathrm{C}_{\beta}$ bonds $\left(\chi_{1}\right.$ angles $)$. Notably, the residues where $\chi_{2}$ gets more constrained upon nitration are Tyr48 and Tyr97, which do not seem to experience chemical exchange to the NMR spectra. In addition, the rotation of the aromatic ring of Tyr67 is much allowed, even when the residue is nitrated. This may explain the absence of the signal in the ${ }^{1} \mathrm{H}-{ }^{15} \mathrm{~N}$ HSQC spectra, since this residue dwells on top of the haem porphyrin.

\section{Discussion}

The aim of this work was to develop a reliable methodology for assessing the configuration of nitrated tyrosines within a protein, as well as their dynamic behaviour. For this purpose, we chose the human metalloprotein $\mathrm{C} c$, which displays five tyrosine residues in different molecular environments (see Figure S1, in the Supporting Information). Two of them (Tyr46 and Tyr74) are highly exposed to solvent and two of them (Tyr48 and Tyr97) only partially. The fifth one (Tyr67) is totally buried in the protein. Two of them (Tyr46 and Tyr48) are reported to be nitrated in vitro, ${ }^{[25]}$ but never detected in vivo. Still, both are located in a loop, one of them (Tyr46) is fully exposed to the solvent, and no competition for peroxynitrite is expected except themselves-one lies near the other. In summary, all these residues fulfil the requirements reported by Ischiropoulos. ${ }^{[4]}$

In this work, we have described a non-damaging method to determine the configuration of nitrotyrosine residues of native proteins in solution. This requires performing the nitration with ${ }^{15} \mathrm{~N}$-labelled peroxynitrite, which is easily obtained with $\mathrm{Na}^{15} \mathrm{NO}_{2}$, and optimizing the INEPT delay (20.8 ms) for the detection of the ${ }^{3} J(\mathrm{~N}-\mathrm{H})$ couplings (equal to about $10-12 \mathrm{~Hz}$ ). The ${ }^{1} \mathrm{H}^{13}{ }^{13} \mathrm{C}$ HSQC spectra were obtained under natural abundance conditions to obviate an expensive ${ }^{13} \mathrm{C}$ tag. Using this method, we are able to detect four out of five nitrated residues and further, to determine the configuration of each nitrotyrosine residue in the modified proteins and their different dynamic behaviour in the protein environment. In addition, our approach allows reliable data on the dynamics of the nitrated residue to be obtained. Two circumstances concur for the residue whose nitration is not detected: the aromatic ring lies near the haem moiety and it shows a dynamic behaviour, according to our MD computations. This may make its ring protons undergo drastic perturbations, thereby inducing NMR line-broadening beyond detection.

\section{Conclusion}

A fast, suitable and non-damaging method for the analysis of nitrotyrosine residues and their dynamics in native proteins has been developed. Major outcomes can be expected from the broad applicability of this methodology and the relevance of protein nitration with regards to oxidative stress response, cell signalling and disease development.

\section{Experimental Section}

Sample preparation: Recombinant monotyrosine mutants of human respiratory $\mathrm{C} c(h-\mathrm{C} c)$ were expressed in the $E$. coli $\mathrm{DH} 5 \alpha$ strain and further purified by ionic exchange chromatography, as previously described. ${ }^{[1,26]}$ The synthesis of peroxynitrite was as reported previously, ${ }^{[14]}$ by using $\mathrm{Na}^{15} \mathrm{NO}_{2}$ (Cambridge Isotope Laboratories, Inc., USA) as a substrate. Nitration of monotyrosine $\mathrm{C} c$ mutants were also performed as previously described, ${ }^{[11,14]}$ with minor modifications. ${ }^{[16]}$ Therefore, Fe ${ }^{\mathrm{III}}$-EDTA concentration and the number of additions of peroxynitrite were increased up to $1.5 \mathrm{~mm}$ and ten bolus additions, respectively. Also, $\mathrm{C} c$ was nitrated under acidic conditions ( $\mathrm{pH}$ 5). Afterwards, the resulting nitrated $\mathrm{C} c$ species were intensively washed in $10 \mathrm{~mm}$ potassium phosphate at $\mathrm{pH} 6$ and purified as reported previously. ${ }^{[11,16]}$ The purity to homogeneity of nitrated $\mathrm{C} c$ preparations, the molecular mass and the specific nitrated tyrosine of each mutant were confirmed by tryptic digestion and MALDI-TOF (Bruker-Daltonics, Germany) analyses. Actually, nitrated Cc preparations were purified to $95 \%$ homogeneity. Western Blotting Solution (Amersham) with antibodies anti-nitrotyrosine (Biotem) corroborated the presence of the $-\mathrm{NO}_{2}$ group in the $\mathrm{C} c$ samples upon nitration. Samples were concentrated to $0.2-2.0 \mathrm{~mm}$ in sodium phosphate buffer ( $5 \mathrm{~mm}, \mathrm{pH} 6)$.

NMR spectroscopy: ${ }^{1} \mathrm{H}-{ }^{15} \mathrm{~N}$ HSQC $2 \mathrm{D}$ experiments on ${ }^{15} \mathrm{~N}$-labeled nitrated samples were performed on a Bruker Digital Avance $900 \mathrm{MHz}$ NMR spectrometer provided with a TXI triple resonance cryoprobe (at the Centre of Magnetic Resonances, CERM, of the University of Florence). The INEPT ${ }^{[23]}$ delay $(20.8 \mathrm{~ms})$ was optimized for the detection of the ${ }^{3} J(\mathrm{~N}-\mathrm{H})$ couplings (equal to about $10-12 \mathrm{~Hz}$ ). The spectra were acquired with spectral windows of $\delta=16 \mathrm{ppm}$ on the ${ }^{1} \mathrm{H}$ and $\delta=150 \mathrm{ppm}$ on the ${ }^{15} \mathrm{~N}$. The later window was centred on $\delta=350 \mathrm{ppm}$, since the chemical shift $(\delta)$ of $\mathrm{TyrNO}_{2}$ is approximately $370 \mathrm{ppm} \cdot{ }^{[27]}{ }^{1} \mathrm{H}-{ }^{13} \mathrm{C}$ HSQC $2 \mathrm{D}$ sensitivity-enhanced ${ }^{[28]}$ experiments on natural-abundance $-{ }^{13} \mathrm{C}$ samples were performed on a Bruker Digital Avance $600 \mathrm{MHz}$ NMR spectrometer 
equipped with a TCI triple resonance cryoprobe. Sample concentrations were $1 \mathrm{~mm}$, for untreated species and ranged from 0.2 to $0.9 \mathrm{~mm}$ for the nitrated forms of the distinct single-tyrosine mutants. All the samples were reduced with equimolar amounts of sodium ascorbate just before recording the spectra, excepting nitrated and non-nitrated $\mathrm{C} c$ monotyrosine 46 and 48 mutants.

Molecular Dynamics Computations: Molecular Dynamics (MD) simulations were carried out using AMBER 9.0 ${ }^{[29]}$ under the AMBER 96 force field. Force-field parameters for the haem moiety were those reckoned by Autenrieth. ${ }^{[30]}$ All calculations were run under periodic boundary conditions using an orthorhombic (minimum distance between protein and cell faces, initially set to $10 \AA$ ) cell geometry and PME electrostatics with a Ewald summation-cut-off of $9 \AA$. Chloride counterions were added to neutralize charges. All systems were solvated with TIP3P water molecules. ${ }^{[31]}$ Protein side-chains were then energy-minimized. Afterwards, solvent and counter-ions were subjected to 500 steps of energy minimization and then submitted to 300 ps NPT-MD using isotropic molecule position scaling and a pressure relaxation time of 2 ps at $298 \mathrm{~K}$. Temperature was regulated with Berendsen's heat bath algorithm ${ }^{[32]}$ using a coupling time constant equal to $0.5 \mathrm{ps}$. Then, for each protein, the whole system was energy minimized and submitted to NVT-MD at $298 \mathrm{~K}$, using 2 fs integration time steps. Snapshots were saved every 1 ps. The SHAKE algorithm ${ }^{[33]}$ was used to constrain bonds involving hydrogen atoms. Coordinate files were processed using the PTRAJ module of AMBER.

\section{Acknowledgements}

This work was supported by the Spanish Ministry of Science and Innovation (BFU2006-01361/BMC and BFU2009-07190). Authors thank financial support in the form of Access to the Bio-NMR Research Infrastructure co-funded under the 7th Framework Programme of the EC (FP7/ 2007-2013) grant agreement 261863 and project contract RII3-026145 and LRB access grants (ICTS 2685-10 and 2661-10).

[1] P. Pacher, J. S. Beckman, L. Liaudet, Physiol. Rev. 2007, 87, 315424.

[2] H. Ischiropoulos, Biochem. Biophys. Res. Commun. 2003, 305, 776783.

[3] T. Koeck, X. Fu, S. L. Hazen, J. W. Crabb, D. J. Stuehr, K. S. Aulak, J. Biol. Chem. 2004, 279, 27257-27262.

[4] H. Ischiropoulos, Arch. Biochem. Biophys. 2009, 484, 117-121.

[5] J. M. Souza, G. Peluffo, R. Radi, Free Radical Biol. Med. 2008, 45, 357-366.

[6] J. M. García-Heredia, A. Díaz-Quintana, M. Salzano, M. Orzáez, E. Pérez-Payá, M. Teixeira, M. A. De La Rosa, I. Díaz-Moreno, J. Biol. Inorg. Chem. 2011, 16, 1155-1168.

[7] J. M. Souza, I. Choi, Q. Chen, M. Weisse, E. Daikhin, M. Yudkoff, M. Obin, J. Ara, J. Horwitz, H. Ischiropoulos, Arch. Biochem. Biophys. 2000, 380, 360-366.

[8] J. M. Souza, E. Daikhin, M. Yudkoff, C. S. Raman, H. Ischiropoulos, Arch. Biochem. Biophys. 1999, 371, 169-178.

[9] N. Abello, H. A. Kerstjens, D. S. Postma, R. Bischoff, J. Proteome Res. 2009, 8, 3222-3238.

[10] C. Quijano, D. Hernandez-Saavedra, L. Castro, J. M. McCord, B. A. Freeman, R. Radi, J. Biol. Chem. 2001, 276, 11631-11638.

[11] V. Rodriguez-Roldan, J. M. Garcia-Heredia, J. A. Navarro, M. A. De La Rosa, M. Hervas, Biochemistry 2008, 47, 12371-12379.
[12] N. Romero, R. Radi, E. Linares, O. Augusto, C. D. Detweiler, R. P. Mason, A. Denicola, J. Biol. Chem. 2003, 278, 44049-44057.

[13] L. A. Abriata, A. Cassina, V. Tortora, M. Marin, J. M. Souza, L. Castro, A. J. Vila, R. Radi, J. Biol. Chem. 2009, 284, 17-26.

[14] A. M. Cassina, R. Hodara, J. M. Souza, L. Thomson, L. Castro, H. Ischiropoulos, B. A. Freeman, R. Radi, J. Biol. Chem. 2000, 275, 21409-21415.

[15] V. Demicheli, C. Quijano, B. Alvarez, R. Radi, Free Radical Biol. Med. 2007, 42, 1359-1368.

[16] J. M. García-Heredia, I. Diaz-Moreno, P. M. Nieto, M. Orzaez, S. Kocanis, M. Teixeira, E. Perez-Paya, A. Diaz-Quintana, M. A. De La Rosa, Biochim. Biophys. Acta Bioenerg. 2010, 1797, 981-993.

[17] H. J. Lucas, Y. P. Liu, J. Am. Chem. Soc. 1933, 55, 1271-1280.

[18] H. Ohshima, M. Friesen, I. Brouet, H. Bartsch, Food Chem. Toxicol. 1990, 28, 647-652.

[19] H. Yang, Y. Zhang, U. Pöschl, Anal. Bioanal. Chem. 2010, 397, 879886.

[20] B. Ghesquière, K. Helsens, J. Vandekerckhove, K. Gevaert, Proteomics 2011, 11, 1094-1098.

[21] S. A. Greenacre, H. Ischiropoulos, Free Radical Res. 2001, 34, 541 581.

[22] M. Sokolovsky, J. F. Riordan, B. L. Vallee, Biochem. Biophys. Res. Commun. 1967, 27, 20-25.

[23] G. A. Morris, J. Am. Chem. Soc. 1980, 102, 428-429.

[24] H. Nakagawa, N. Komai, M. Takusagawa, Y. Miura, T. Toda, N. Miyata, T. Ozawa, N. Ikota, Biol. Pharm. Bull. 2007, 30, 15-20.

[25] I. Díaz-Moreno, J. M. García-Heredia, A. Díaz-Quintana, M. Teixeira, M. A. De La Rosa, Biochim. Biophys. Acta 2011, 1807, 16161623.

[26] V. Rodríguez-Roldán, J. M. García-Heredia, J. A. Navarro, M. Hervás, B. De La Cerda, F. P. Molina-Heredia, M. A. De La Rosa, Biochem. Biophys. Res. Commun. 2006, 346, 1108-1113.

[27] W. J. Skawinski, F. Adebodun, J. T. Cheng, F. Jordan, R. Mendelsohn, Biochim. Biophys. Acta Protein Struct. Mol. Enzymol. 1993 1162, 297-308.

[28] A. G. Palmer III, J. Cavanagh, P. E. Wright, M. Rance, J. Magn Reson. 1991, 93, 151-170.

[29] D. A. Case, T. A. Darden, T. E. Cheatham III, C. L. Simmerling, J Wang, R. E. Duke, R. Luo, K. M. Merz, D. A. Pearlman, M. Crowley, R. C. Walker, W. Zhang, B. Wang, S. Hayyik, A. Roitberg, G Seabra, K. F. Wong, F. Paesani, X. Wu, S. Brozell, V. Tsui, H. Gohlke, L. Yang, C. Tan, J. Mongan, V. Hornak, G. Cui, P. Beroza D. H. Mathews, C. Schafmeister, W. S. Ross, P. A. Kollman, AMBER 9, University of California, San Francisco, 2006.

[30] F. Autenrieth, E. Tajkhorshid, J. Baudry, Z. Luthey-Schulten, J. Comput. Chem. 2004, 25, 1613-1622.

[31] W. L. Jorgensen, J. Chandrasekhar, J. D. Madura, R. W. Impey, M. L. Klein, J. Chem. Phys. 1983, 79, 926-935.

[32] H. J. C. Berendsen, J. P. M. Postma, W. F. Vangunsteren, A. Dinola, J. R. Haak, J. Chem. Phys. 1984, 81, 3684-3690.

[33] J. P. Ryckaert, G. Ciccotti, H. J. C. Berendsen, J. Comput. Phys. 1977, 23, 327-341.

[34] P. E. Hansen, Org. Magn. Res. 1979, 12, 109-142.

[35] W.-Y. Jeng, C.-Y. Chen, H.-C. Chang, W.-J. Chuang, J. Bioenerg. Biomembr. 2002, 34, 423-431.

[36] J. P. Crow, H. Ischiropoulos, Nitric Oxide Part B 1996, 269, 185-194.

[37] J. Mason. Nitrogen, in: J. Mason (Ed.), Multinuclear NMR, Plenum Press, London, 1987, pp. 335-365.

Received: October 31, 2011 Published online: February 29, 2012 